\title{
Linkage with cathepsin B-sensitive dipeptide promotes the in vitro and in vivo anticancer activity of PEGylated tumor necrosis factor-alpha (TNF- $\alpha$ ) against murine fibrosarcoma
}

\author{
DAI ChuanYun ${ }^{1,2}$, FU Ya ${ }^{2}$, LI Biao $^{3}$, WANG YiGuang ${ }^{1}$, ZHANG Xuan ${ }^{1}$, \\ WANG JianCheng ${ }^{1} \&$ ZHANG Qiang ${ }^{1 *}$ \\ ${ }^{1}$ State Key Laboratory of Natural and Biomimetic Drugs, School of Pharmaceutical Sciences, Peking University, Beijing 100191, China; \\ ${ }^{2}$ Department of Biology, Chongqing University of Science and Technology, Chongqing 401331, China; \\ ${ }^{3}$ Institute of Medicinal Plant Development, Chinese Academy of Medical Sciences \& Peking Union Medical College, Beijing 100193, China
}

Received November 11, 2010; accepted December 5, 2010

\begin{abstract}
To improve the pharmacological profile of tumor necrosis factor alpha (TNF- $\alpha$ ), we have synthesized a new PEGylated prodrug, PEG-vcTNF- $\alpha$, using a cathepsin B-sensitive dipeptide (valine-citrulline, vc) to link branched PEG and TNF- $\alpha$. PEG-modified TNF- $\alpha$ without the dipeptide linker (PEG-TNF- $\alpha$ ) and unconjugated TNF- $\alpha$ were also tested as controls. It was found for the first time that TNF- $\alpha$ released from PEG-vcTNF- $\alpha$ was specifically dependent on the presence of cathepsin B. PEG-vcTNF- $\alpha$ induced higher cytotoxicity and greater apoptosis against L929 murine fibrosarcoma cells than PEG-TNF- $\alpha$. Reversal of these effects by a cathepsin-B inhibitor confirmed that these effects were mediated by cathepsin B-specific release of TNF- $\alpha$. In vivo pharmacokinetics studies demonstrated that the plasma stability of PEG-vcTNF- $\alpha$ was significantly increased compared to TNF- $\alpha$. Finally, the improved anticancer efficacy of PEG-vcTNF- $\alpha$ and the distinct activities among the three formulations confirmed the positive contribution of both PEGylation and the dipeptide linkage to the improved drug-like properties of PEG-vcTNF- $\alpha$. The results here indicate that linking proteins and PEG via the cathepsin B-sensitive dipeptide may be a promising strategy for developing protein therapeutics.
\end{abstract}

tumor necrosis factor alpha, PEGylation, cathepsin B-sensitive dipeptide, antitumor effect

Citation: Dai C Y, Fu Y, Li B, et al. Linkage with cathepsin B-sensitive dipeptide promotes the in vitro and in vivo anticancer activity of PEGylated tumor necrosis factor-alpha (TNF- $\alpha$ ) against murine fibrosarcoma. Sci China Life Sci, 2011, 54: 128-138, doi: 10.1007/s11427-010-4124-z

Tumor necrosis factor- $\alpha$ (TNF- $\alpha)$, a cytokine secreted by activated macrophages, was first described as a factor present in infected, endotoxin-injected mice that caused hemorrhagic necrosis of transplanted Math-A fibrosarcoma [1]. TNF- $\alpha$ has a direct anti-tumor effect, and stimulates host anti-tumor immune responses via specific interactions with tumor vascular endothelial cells [2]. TNF- $\alpha$ can bind to TNF- $\alpha$ receptors (the $55 \mathrm{kD}$ TNF- $\alpha \mathrm{R} 1$ and the $75 \mathrm{kD}$ TNF- $\alpha$ R2), which are located on the plasma membrane of most cells throughout the body except red blood cells. Act-

*Corresponding author (email: zqdodo@bjmu.edu.cn) ing via these receptors, TNF- $\alpha$ induces two main forms of "extrinsic" (or death receptor-mediated) programmed cell death (PCD): (i) classical apoptosis, characterized by caspase-dependent chromatin condensation and fragmentation, membrane blebbing and generation of apoptotic bodies; and (ii) necrosis-like caspase-independent PCD, characterized by absent or marginal chromatin condensation, lack of nuclear fragmentation and disruption of membrane integrity [3]. Thus TNF- $\alpha$ has been considered as a promising new therapeutic agent $[4,5]$. However, it is cleared rapidly from the circulation and widely distributed to various tissues after intravenous administration, resulting in limited 
bioavailability. Although systemic administration of TNF- $\alpha$ causes the regression of various transplanted solid tumors [6], the high doses required for significant clinical anti-tumor effects often also elicit unexpected side-effects, such as tissue inflammation and injury, as well as the lethal endotoxic shock-like syndrome [7,8]. Similar in vivo drawbacks to those seen with TNF- $\alpha$ are also found in clinical applications of other bioactive proteins [9]. Thus, the development of a drug delivery system (DDS) seems necessary for the promotion of protein therapies, following recent marked advances in biotechnology-based pharmaceuticals.

In recent years, PEGylation has been identified as one of the most promising strategies for optimizing protein therapeutics [10]. The attachment of polyethylene glycol (PEG) to bioactive proteins (PEGylation) increases their molecular size and steric hindrance, leading to increased plasma half-life and in vivo stability. However, protein PEGylation is mostly non-specific and occurs at all the lysine residues of the protein, some of which may be in or near an active site. Furthermore, most PEG conjugates may not be cleavable in vivo. As a result, protein PEGylation is usually accompanied by a significant loss of bioactivity because of the loss of a binding site and/or steric hindrance. For instance, $\mathrm{BPEG}_{40 \mathrm{k}}$-IFN- $\alpha 2 \mathrm{a}$, a mixture of various positional isomers of interferon- $\alpha 2$ a randomly conjugated with $40 \mathrm{kD}$ branched PEG, lost approximately $90 \%$ of its bioactivity compared to unmodified IFN- $\alpha 2 \mathrm{a}[11,12]$. Thus, the clinical application of PEGylated proteins has been limited.

To overcome the problem of PEGylation, some investigators have attempted replacing all lysine residues in the protein with other amino acid residues. This lysine-deficient protein was then site-specifically mono-PEGylated at its N-terminus. For instance, site-specific PEGylation of TNF- $\alpha[13,14]$ and human basic fibroblast growth factor [15] has been reported. Nevertheless, the slow rate of free drug release from the conjugate strongly influenced the in vivo antitumor activity of these PEGylated proteins. However, it is logical to hypothesize that i.v. administration of a TNF- $\alpha$ contained system in which the PEG and TNF- $\alpha$ are linked by an oligopeptide that is the substrate of a tumor tissue-specific protease may lead to high anticancer activity, even at low doses, because of the combined effect of extended circulation time and tumor site-specific drug release.

Cathepsin B is a ubiquitous cysteine protease whose properties differ very little between species [16,17]. It is never found in extracellular areas, except in pathological conditions such as tumors or in areas of tissue destruction in rheumatoid arthritis [18]. Therefore, the cathepsin B-sensitive peptide is likely to be stable in circulation. The physiological substrates of cathepsin B and L include valine-citrulline and phenylalanine-arginine [19]. Previous reports have demonstrated the success of conjugating a cytotoxic agent to a tumor-selective monoclonal antibody $(\mathrm{mAb})$ using a protease-sensitive dipeptide linker. These antibody-drug conju- gates include cBR96-valine-citrulline-MMAE and BR96valine-citrulline-DOX (specific to Lewis $\mathrm{Y}$ antigen on carcinomas), cAC10-valine-citrulline-MMAE (specific to CD30 on hematological malignancies), and huPr1-valine-citrullineMMAE (specific to hormone-resistant prostate cancer), and have high stability in plasma and efficient release of drug in tumor tissues [20-25].

In the present study, we have designed a novel PEGylated protein using a cathepsin B-cleavable dipeptide linker, valine-citrulline, to link the PEG and TNF- $\alpha$, to overcome the short half-life and poor tissue selectivity of unconjugated TNF- $\alpha$ and the low therapeutic efficacy of standard PEGylated TNF- $\alpha$ (PEG-TNF- $\alpha$ ). This validation study of this novel antitumor prodrug, PEG-valine-citrulline-TNF- $\alpha$ (PEG-vcTNF- $\alpha$ ), includes assessment of the specific bioactivities, stability, sensitivity to cathepsin $\mathrm{B}$, in vitro cytotoxicity and apoptosis, pharmacokinetics, and antitumor activity, compared with the standard PEGylated TNF- $\alpha$ or unconjugated TNF- $\alpha$, in the hope that we can prove the hypothesis mentioned above.

\section{Materials and methods}

\subsection{Materials}

Recombinant human tumor necrosis factor- $\alpha$ (rhTNF- $\alpha$, $17000 \mathrm{kD}, 1 \times 10^{7} \mathrm{IU} \mathrm{mg}^{-1} \mathrm{TNF}-\alpha$ in $0.2 \mathrm{~mol} \mathrm{~L}^{-1}$ phosphate buffer, $\mathrm{pH}$ 7.2) was obtained from Biosynthesis Biotechnology Co., Ltd. (Beijing, China); TNF- $\alpha$ ELISA Detection Kit was from Senxiong Technical Industry Co, Ltd. (Shanghai, China); $40 \mathrm{kD}$ branched PEG-NHS (Polydispersity, 1.05 ) and $40 \mathrm{kD}$ branched PEG-val-cit-NHS (Polydispersity, 1.05) were supplied from Jenkem Technology Co., Ltd. (Beijing, China); Cathepsin B from human liver (2759 units $\mathrm{mg}^{-1}$ protein) was purchased from Sigma Corporation; L929 murine fibrosarcoma cells lines were from Life Technologies (Gaithersburg, MD) and were grown in RPMI 1640 medium supplemented with $10 \%$ fetal bovine serum. Annexin V-FITC Apoptosis Detection Kits and FITC were obtained from Biosea Biotechnology Co., Ltd. (Beijing, China) and Cell Counting Kits (CCK-8) were obtained from Dojindo Laboratories (Kumamoto, Japan).

\subsection{Preparation and characterization of TNF- $\alpha$ conju- gates}

Branched $40 \mathrm{kD}$ PEG-NHS or PEG-vc-NHS moiety was added at room temperature and under rapid stirring in $0.1 \mathrm{~mol} \mathrm{~L}^{-1}$ phosphate buffer, $\mathrm{pH}$ 8.5. For these experiments, recombinant human TNF- $\alpha$ was reacted with a 30 -fold molar excess of activated branched PEG moiety. The conjugation reaction was carried out for $30 \mathrm{~min}$, while the $\mathrm{pH}$ was maintained with $0.05 \mathrm{~mol} \mathrm{~L}^{-1} \mathrm{NaOH}$. Subsequently, 6-ami- 
nocaproic acid (5-fold molar excess against activated branched PEG moiety) was added to stop the reaction and the $\mathrm{pH}$ was adjusted to 6.0 with $0.1 \mathrm{~mol} \mathrm{~L}^{-1} \mathrm{HCl}$. The synthesized PEG-TNF- $\alpha$ or PEG-vcTNF- $\alpha$ conjugates were purified from the unreacted polymer and the side reaction products by gel filtration chromatography using a Sephacryl S-200HR column on a FPLC system eluted with $0.1 \mathrm{~mol} \mathrm{~L}^{-1}$ phosphate buffer/0.3 mol L ${ }^{-1} \mathrm{NaCl}, \mathrm{pH}$ 7.0. The molecular size of PEG-TNF- $\alpha$ or PEG-vcTNF- $\alpha$ conjugates was estimated by analytical gel filtration chromatography using a Sephacryl S-300HR column, eluted with $10 \mathrm{~mol} \mathrm{~L}^{-1}$ phosphate buffer/0.3 mol L ${ }^{-1} \mathrm{NaCl}, \mathrm{pH} 7.0$. and the degree of modification by PEG attachment to TNF- $\alpha$ was assayed by fluorescamine methods as described by Stocks et al. [26]. The in vitro specific bioactivity of PEG-TNF- $\alpha$ or PEGvcTNF- $\alpha$ conjugates was measured by L-M cytotoxicity assay, according to the method described by Yamazaki et al. [27].

\subsection{Stability of TNF- $\alpha$ conjugates in plasma}

To evaluate stability of PEG-TNF- $\alpha$ and PEG-vcTNF- $\alpha$ in plasma, amounts of PEG-TNF- $\alpha$ or PEG-vcTNF- $\alpha$ conjugates were labeled with FITC yielding PEG-TNF- $\alpha$-FITC or PEG-vcTNF- $\alpha$-FITC conjugates [28]. Conjugates containing $17 \mu \mathrm{g}$ of protein in each sample were incubated in $1 \mathrm{~mL}$ of freshly drawn mouse or human plasma pre-incubated at $37^{\circ} \mathrm{C}$. Aliquots of $100 \mu \mathrm{L}$ were sampled periodically. All samples were lyophilized immediately, then dissolved in $1 \mathrm{~mL}$ chloroform, vortexed for $10 \mathrm{~min}$, and centrifuged at $5000 \times g$ for $5 \mathrm{~min}$. The upper layer was collected and chloroform was removed by lyophilization. The resulting extracted PEG mixture was renewably dissolved in 1 $\mathrm{mL}$ phosphate-buffered saline (PBS) and analyzed by a fluorescence spectrophotometer $\left(\lambda_{\mathrm{ex}}=492 \mathrm{~nm}, \lambda_{\mathrm{em}}=519 \mathrm{~nm}\right)$. This result was then converted into the TNF- $\alpha$ concentration (validation data of assay not shown).

\subsection{Purification of lysosomes}

Sprague-Dawley rats (200-300 g) were sacrificed to harvest and purify their liver lysosomes. Lysosomes were collected from liver homogenate by centrifugation using the metrizamide discontinuous gradient method with a slight modification [29]. Briefly, non-fasted rats were anesthetized with ether and blooded. The liver was removed and homogenized using a Polytron mixer (Kinematica, Switzerland). The homogenate ( $4 \mathrm{~mL}$ of chilled $0.25 \mathrm{~mol} \mathrm{~L}^{-1}$ sucrose/g of liver) was centrifuged in succession at $4800 \times g$ for $5 \mathrm{~min}$, and $17000 \times g$ for $10 \mathrm{~min}$. The sediment of the second centrifugation was washed at $17000 \times g$ for $10 \mathrm{~min}$, resuspended in $57 \%$ metrizamide, and $10 \mathrm{~mL}$ thereof was placed on the bottom of an Ultraclear tube (Beckman, CA). On top, a discontinuous gradient of metrizamide was constructed (layers from bottom to top were: $57 \%, 32.8 \%, 26.3 \%$, and $19.8 \%$ metrizamide). Centrifugation was for $1 \mathrm{~h}$ in a $70.1 \mathrm{Ti}$ rotor (Beckman) at $141000 \times g$. Lysosomes were collected from the top layer and from the 26.3/19.8 interface. Using this procedure, the degree of purification of lysosomes relative to the original homogenate (as measured by acid phosphatase activity) was 20-30-fold and the yield 3\%-5\%. The protein concentrations in lysosomal pellets were determined by the BCA assay (Pierce, Rockford, IL). Acid phosphatase activity was determined using $\beta$-glycerophosphate or $\rho$-nitrowith phenyl phosphate as the substrate. The activities of cathepsin B and lysosomes were determined by using Z-Arg-Arg-AMC as the substrate.

\subsection{In vitro evaluation of drug release}

All release studies were carried out at $37^{\circ} \mathrm{C}$. The concentrations of conjugates labeled with FITC in the incubation mixture were approximately $20 \mu \mathrm{g} \mathrm{mL}^{-1}$, corresponding to around $17 \mu \mathrm{g} \mathrm{mL} \mathrm{m}^{-1}$ of TNF- $\alpha$. Four in vitro testing protocols were performed. First, the hydrolysis stability of TNF- $\alpha$ conjugates to the liver lysosomes or homogenates was assessed. TNF- $\alpha$ conjugates were incubated in $50 \mathrm{mmol} \mathrm{L}{ }^{-1}$ sodium acetate ( $\mathrm{pH} 5.0), 2 \mathrm{mmol} \mathrm{L}^{-1}$ dithiothreitol (DTT), and $25 \%(\mathrm{v} / \mathrm{v})$ glycerol for $15 \mathrm{~min}$ at $37^{\circ} \mathrm{C}$ and then added as a $20 \%(\mathrm{v} / \mathrm{v})$ addition to initiate each reaction [26]. The final concentrations of lysosomes and homogenates were 1 $\mathrm{mg}$ of protein $\mathrm{mL}^{-1}$ and $20 \% \mathrm{w} / \mathrm{w}$, respectively. Second, we studied the hydrolysis stability of TNF- $\alpha$ conjugates in the presence of various types of pure enzymes at concentrations of approximately $8 \mu \mathrm{mol} \mathrm{L}{ }^{-1}$. This panel of enzymes included include serine proteases (trypsin and chymotrypsin), cysteine proteases (papain and cathepsin B), aspartic acid proteases (cathepsin D and pepsin A), and metalloproteases (thermolysin and collagenase). Also, to examine the effect of $\mathrm{pH}$ on drug release, acetate or phosphate buffers (40 mmol $\mathrm{L}^{-1}$ ), with a $\mathrm{pH}$ range from 3 to 7 , were used. In the case of serine or metalloproteases, a final concentration of $10 \mathrm{mmol} \mathrm{L}^{-1} \mathrm{CaCl}_{2}$ was added, while in the case of cysteine proteases, final concentrations of $1 \mathrm{mmol} \mathrm{L}^{-1}$ EDTA and 5 mmol L ${ }^{-1}$ reduced glutathione were added. Third, the effect of several enzyme inhibitors was evaluated. They include aprotinin, bestatin, pepstatin, phosphoramidon, E-64, Z-Phe-Phe- $\mathrm{CHN}_{2}$, and CA-074. Conjugate-lysosome mixtures were as described above, but contained a final inhibitor concentration of $10 \mu \mathrm{mol} \mathrm{L}{ }^{-1}$. Aliquots of $100 \mu \mathrm{L}$ were sampled periodically, and all samples were treated with the same methods described above (in section "Stability of TNF- $\alpha$ conjugates in plasma").

\subsection{In vitro cytotoxicity}

Cells $\left(2 \times 10^{4}\right.$ cells well ${ }^{-1}$ in $100 \mu \mathrm{L}$ medium $)$ were placed into wells of a 96-well microtiter plate. After $24 \mathrm{~h}$ incuba- 
tion, TNF- $\alpha$, PEG-TNF- $\alpha$, PEG-vcTNF- $\alpha$, cathepsin B (final concentration, 10 units $\mathrm{mL}^{-1}$ ), CA-074 (final concentration, $10 \mu \mathrm{mol} \mathrm{L} \mathrm{L}^{-1}$ ) or PBS was added to the wells according to experiment design. The volume was adjusted by culture medium to $200 \mu \mathrm{L}$, and the incubation was carried out. Cell viability assessment was performed by using Cell Counting Kits (CCK-8), following the manufacturer's instructions. Cytotoxicity $(\mathrm{C} \%)$ was calculated from the equation $\mathrm{C}=(\mathrm{A}-\mathrm{B}) / \mathrm{A} \times 100 \%$, where $\mathrm{A}$ is the absorption in a control well and $\mathrm{B}$ is the absorption in the test well. L929 cells were placed in wells $\left(1 \times 10^{6}\right.$ cells well $\left.^{-1}\right)$ and incubated for $24 \mathrm{~h}$ prior to adding TNF- $\alpha$ (10 IU $\left.\mathrm{mL}^{-1}\right)$, PEG-TNF- $\alpha$ or PEG-vcTNF- $\alpha$. In the latter two conditions (PEG-TNF- $\alpha$ or PEG-vcTNF- $\alpha$ ), $10 \mathrm{IU} \mathrm{mL}^{-1}$ TNF- $\alpha$ was also included. Cells were incubated for a further $12 \mathrm{~h}$. The culture medium was discarded and replaced with $100 \mu \mathrm{L}$ of cold binding buffer. Cell staining was performed using Annexin V-FITC according to the instructions of the manufacturer. Samples were analyzed using a FACS Canto (BD, USA) flow cytometer.

\section{7 vivo study on antitumor effects}

All of the animal experiments adhered to the principles of care and use of laboratory animals and were approved by the Institutional Animal Care and Use Committee of Peking University. The antitumor effects of TNF- $\alpha$ were assessed in mice bearing Meth-A fibrosarcoma. The Meth-A cells were implanted intradermally $\left(2 \times 10^{5}\right.$ cells per site $)$ in 5 -week-old female BALB/c mice. Seven days later (when tumor diameter was $~ 7-8 \mathrm{~mm}$ ), native TNF- $\alpha$, PEG-TNF- $\alpha$, and PEG-vcTNF- $\alpha$ were administered by a single intravenous injection. The antitumor potency was estimated from the tumor volume and tumor hemorrhagic necrosis $24 \mathrm{~h}$ after the injection. Antitumor effects of TNF- $\alpha$ in vivo were screened by determining mean tumor volume (V) as calculated using the formula: $\mathrm{V}=\left(\right.$ length $\left.\times(\text { width })^{2}\right) / 2$.

\subsection{Pharmacokinetics studies in mice}

Native TNF- $\alpha$, PEG-TNF- $\alpha$, and PEG-vcTNF- $\alpha$ were radiolabeled with ${ }^{125} \mathrm{I}$ by the lactoperoxidase method, yielding ${ }^{125}$ I-TNF- $\alpha,{ }^{125}$ I-PEG-TNF- $\alpha$, and ${ }^{125}$ I-PEG-vcTNF- $\alpha$ with specific activities of $4.50 \mathrm{mCi} \mathrm{mg}^{-1}$ protein. The biological activities of ${ }^{125}$ I-radiolabeled TNF- $\alpha$ and its derivatives were indistinguishable from those of non-radiolabeled TNF- $\alpha$ and its derivatives (data not shown). Their pharmacokinetic profiles in blood circulation after i.v. injection into normal male BALB/c mice (5 weeks of age) were studied at a dose of $31.6 \mathrm{ng}$ of protein per mouse. Blood was collected from the tail vein at various time points, and radioactivity was measured in each sample.

\subsection{Statistical analysis}

All data are shown as mean $\pm \mathrm{SD}$, unless specified otherwise. Statistical evaluation was performed with Student's unpaired $t$-test and one-way analysis of variance.

\section{Results}

\subsection{Preparation of conjugates}

Recombinant human TNF- $\alpha$ was conjugated with activated PEG (average molecular weight (MW) 40000; MW/average MW, 1.05) via amide bonds between amino groups of TNF- $\alpha$ and $N$-hydroxysuccinimide groups of PEG at the end of the main chain. The rate of PEG attachment to TNF- $\alpha$ increased with increasing reaction time (data not shown). The resulting PEGylated TNF- $\alpha$ conjugates were purified from native TNF- $\alpha$ and separated into three fractions of various molecular sizes by gel filtration-high performance liquid chromatography (protein standard). Table 1 shows the average MW, degree of PEG modification, and the activities of native TNF- $\alpha$, PEG-TNF- $\alpha$ and PEGvcTNF- $\alpha$. It was demonstrated that activity decreased with

Table 1 Comparison of molecular properties of TNF- $\alpha$, PEG-TNF- $\alpha$ and PEG-vcTNF- $\alpha$ ( $n=5$, mean \pm SD)

\begin{tabular}{|c|c|c|c|c|c|c|}
\hline & Fraction no. & $\begin{array}{l}\text { Average molecu- } \\
\text { lar weight }{ }^{\mathrm{a})}\end{array}$ & $\begin{array}{l}\text { Degree of modifi- } \\
\text { cation }(\%)^{\text {b) }}\end{array}$ & $\begin{array}{c}\text { Specific activity }\left(\times 10^{5} \mathrm{IU} \mathrm{mg}^{-1}\right. \\
\text { TNF- } \alpha)^{\mathrm{c})}\end{array}$ & $\begin{array}{l}\text { Remaining activity } \\
(\%)\end{array}$ & Yield (\%) \\
\hline \multirow{3}{*}{ PEG-TNF- $\alpha$} & 1 & 137000 & 40.46 & $6.23 \pm 0.56^{* * \#}$ & 6.2 & 31.04 \\
\hline & 2 & 99000 & 27.95 & $18.38 \pm 0.87^{* * \#}$ & 9.4 & 24.55 \\
\hline & 3 & 58000 & 8.21 & $27.40 \pm 0.39^{* * \#}$ & 18.6 & 9.38 \\
\hline \multirow{3}{*}{ PEG-vcTNF- $\alpha$} & 1 & 138000 & 38.34 & $28.74 \pm 0.72^{* *}$ & 28.7 & 30.67 \\
\hline & 2 & 101000 & 27.48 & $46.66 \pm 0.43^{*}$ & 46.7 & 23.73 \\
\hline & 3 & 58000 & 9.03 & $74.19 \pm 0.76^{*}$ & 74.2 & 8.38 \\
\hline TNF- $\alpha$ & & 17000 & 0 & $100.0 \pm 0.21$ & 100.0 & \\
\hline
\end{tabular}

a) The molecular size was determined by gel filtration chromatography (protein standard). b) Degree of modification was calculated by fluorescamine methods. c) The specific activities of native TNF- $\alpha$, PEG-TNF- $\alpha$ and PEG-vcTNF- $\alpha$ were measured by growth inhibition L-M cytotoxic assay. *, $P<0.05$; $* *, P<0.01$ vs. native TNF- $\alpha$. \#<0.05 vs. PEG-vcTNF- $\alpha$ under the same degree of modification. 
increasing molecular weight and extensive PEG modification. The specific activity of PEG-vcTNF- $\alpha$ was noticeably higher than that of PEG-TNF- $\alpha$ with the same degree of PEG modification. Fraction 2 was used for further experiments because it combined a moderate degree of modification with relatively high specific activity.

\subsection{Stability of TNF- $\alpha$ conjugates in plasma}

To further evaluate the stability of the PEG-TNF- $\alpha$ or PEG-vcTNF- $\alpha$, conjugates (containing $2 \mu \mathrm{g}$ TNF- $\alpha$ ) were incubated in human or mouse plasma at $37^{\circ} \mathrm{C}$ for a period of 120 h. Endogenous TNF- $\alpha$ was detected in freshly drawn mouse or human plasma but only in trace amounts (data no shown). The stability characteristics of PEG conjugates in human or mouse plasma at $37^{\circ} \mathrm{C}$ are shown in Figure 1, and it can be seen that the two TNF- $\alpha$ conjugates were very stable in plasma. The half-life $\left(t_{1 / 2}\right)$ of PEG-vcTNF- $\alpha$ in vitro is $129.63 \mathrm{~h}$ in human plasma and $48.73 \mathrm{~h}$ in mouse plasma, respectively, while that of PEG-TNF- $\alpha$ is $170.04 \mathrm{~h}$ in human plasma and $60.18 \mathrm{~h}$ in mouse plasma, respectively. Although the $t_{1 / 2}$ of PEG-TNF- $\alpha$ is longer than that of PEG-vcTNF- $\alpha$, there is no significant difference between these two formulations. We also found that release of drugs from the two TNF- $\alpha$ conjugates was faster in human plasma than in mouse plasma.
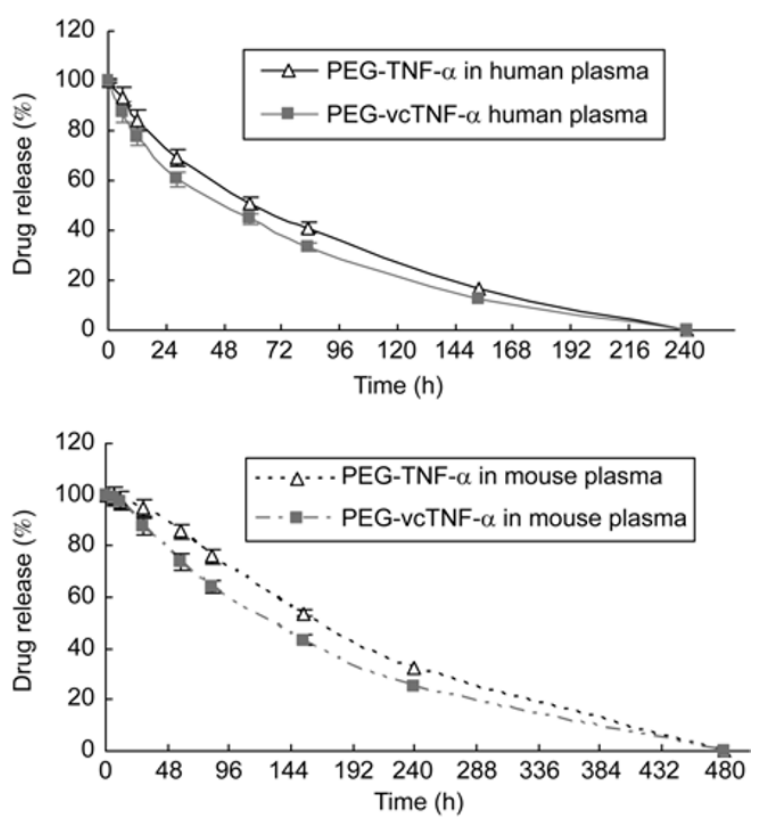

Figure 1 Plasma stability (at $37^{\circ} \mathrm{C}$ ) of conjugated TNF- $\alpha$ constructs (PEG-TNF- $\alpha$ and PEG-vcTNF- $\alpha$ ). PEG-TNF- $\alpha$ and PEG-vcTNF- $\alpha$ conjugates labeled with FITC and containing $17 \mu \mathrm{g}$ of protein in each sample were incubated in $1 \mathrm{~mL}$ of freshly drawn mouse or human plasma pre-incubated at $37^{\circ} \mathrm{C}$. Aliquots of $100 \mu \mathrm{L}$ were sampled at various time points. PEG or PEG conjugates were extracted with chloroform. Fluorescence intensity was analyzed by a fluorescent spectrophotometer $(n=4)$.

\subsection{In vitro evaluation of drug release}

Our focus was on the enzyme specificity of the linker cleavage. First, the excellent stability of TNF- $\alpha$ conjugates in human plasma as well as mouse plasma led us to the hypothesis that lysosomal enzymes are an effective means of TNF- $\alpha$ release. To test this hypothesis, we compared the kinetics of release when TNF- $\alpha$ conjugates were incubated with rat liver homogenates and with lysosomes, prepared using a well-established method for lysosome purification from rat liver. Results showed that the release rate of PEG-vcTNF- $\alpha$ in the liver homogenate was very slow, but was markedly accelerated in the presence of lysosomes, supporting our hypothesis. The $20 \% \mathrm{w} / \mathrm{w}$ homogenate, corresponding to approximately $40 \mathrm{mg} \mathrm{mL}^{-1}$ of protein, was virtually unable to release TNF- $\alpha$, whereas the lysosomes at $1 \mathrm{mg} \mathrm{mL} \mathrm{m}^{-1}$ of protein liberated the drug significantly. Second, to elucidate the enzymes responsible, we measured TNF- $\alpha$ released after $24 \mathrm{~h}$ treatment of TNF- $\alpha$ conjugates with different types of pure enzymes. The results are shown in Table 2, indicating that TNF- $\alpha$ conjugates were very resistant to various kinds of enzymes. However, it was found that cysteine protease was the only enzyme effective in cleaving PEG-vcTNF- $\alpha$, although the release rate varied between papain and bovine spleen cathepsin $\mathrm{B}$ (which was also sensitive to $\mathrm{pH}$ ). PEG-TNF- $\alpha$ was not cleaved at all by any of the enzyme classes (including cysteine protease). Third, to further explore the optimum $\mathrm{pH}$ conditions for drug release, PEG-vcTNF- $\alpha$ was incubated with rat liver lysosomes in buffers covering a $\mathrm{pH}$ range from neutral $(\mathrm{pH}$ 7 ) to acidic ( $\mathrm{pH} 3$ ) for $24 \mathrm{~h}$. Figure 2 shows that the $\mathrm{pH}$ optimum was approximately 4 , identical to that in the case of cathepsin B (Table 2), suggesting that this may be the crucial enzyme in mediating drug release from PEG-vcTNF- $\alpha$.

\subsection{In vitro inhibition studies}

To consolidate these results, we investigated the effect of protease inhibitors. Figure 3 compares the percentages of TNF- $\alpha$ released due to lysosomes in the presence of an inhibitor relative to the control. Inhibitors of amino protease (bestatin), asparatate protease (pepstatin), and metalloprotease (phosphoramidon) were all ineffective at $10 \mu \mathrm{mol} \mathrm{L}^{-1}$. A general inhibitor for cysteine protease (E-64) abolished the release completely, although serine protease inhibitor (aprotinin) also arrested the release to some extent, probably because of its high concentration. This confirms that cysteine proteases are largely responsible for the cleavage of the valine-citrulline linker. Cathepsin B and L are lysosomal cysteine proteases, which play a variety of digestive and processing roles to maintain the normal cellular metabolism [29]. To determine their contributions to the cleavage, similar studies were performed using a specific inhibitor for cathepsin B, CA-074 [30], and for cathepsin L, Z-Phe-Phe- 
Table 2 Effects of enzyme specificity and $\mathrm{pH}$ on drug release after $24 \mathrm{~h}$ incubation of TNF- $\alpha$ conjugates at $37^{\circ} \mathrm{C}$

\begin{tabular}{|c|c|c|c|c|c|c|c|c|c|}
\hline & \multirow{2}{*}{$\mathrm{pH}$} & \multicolumn{2}{|c|}{ Serine proteases } & \multicolumn{2}{|c|}{ Cysteine proteases } & \multicolumn{2}{|c|}{ Aspartate proteases } & \multicolumn{2}{|c|}{ Metalloproteases } \\
\hline & & Trypsin & Chymotrypsin & Papain & Cathepsin B & Cathepsin D & Pepsin A & Thermolysin & Collagenase \\
\hline \multirow{5}{*}{ PEG-vcTNF- $\alpha$} & 3 & 0 & 0 & 22.4 & 10.8 & 0 & 0 & 0 & 0 \\
\hline & 4 & 0 & 0 & 27.0 & 79.6 & 0.4 & 0 & 0 & 0 \\
\hline & 5 & 0 & 0 & 32.6 & 6.7 & 0 & 0.1 & 1.7 & 0 \\
\hline & 6 & 0 & 0 & 27.3 & 1.5 & 0 & 0 & 1.4 & 0.2 \\
\hline & 7 & 0 & 0 & 20.8 & 1.9 & 0 & 0 & 0 & 0 \\
\hline PEG-TNF- $\alpha$ & 4 & 0 & 0 & 0 & 0 & 0 & 0 & 0 & 0 \\
\hline
\end{tabular}

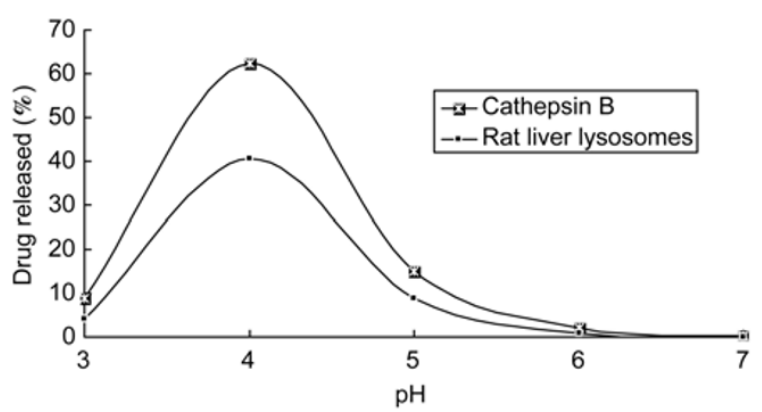

Figure 2 Optimum $\mathrm{pH}$ of enzyme activity for releasing TNF- $\alpha$ from PEG-vcTNF- $\alpha$ labeled with FITC after $24 \mathrm{~h}$ incubation with either 10 units $\mathrm{mL}^{-1}$ cathepsin B or $0.5 \mathrm{mg}$ protein $\mathrm{mL}^{-1}$ of a rat liver lysosome preparation. Conditions: $40 \mathrm{mmol} \mathrm{L}^{-1}$ acetate buffer ( $\left.\mathrm{pH} 3-7\right), 1 \mathrm{mmol} \mathrm{L}^{-1}$ EDTA, $5 \mathrm{mmol} \mathrm{L}^{-1}$ reduced glutathione, $0.1 \%$ Triton X-100; $37^{\circ} \mathrm{C}$.

$\mathrm{CHN}_{2}$ [31]. As shown in Figure 3, both CA-074 and Z-PhePhe- $\mathrm{CHN}_{2}$ were able to dramatically suppress TNF- $\alpha$ re-

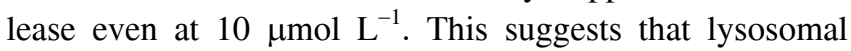
cathepsin $\mathrm{B}$ and cathepsin $\mathrm{L}$ are responsible for the linker cleavage.

\subsection{In vitro cytotoxicity}

As shown in Figure 4, cytotoxicity was reduced in the PEGylated TNF- $\alpha$ constructs, although PEG-vcTNF- $\alpha$ had slightly more toxicity than PEG-TNF- $\alpha$. The $\mathrm{IC}_{50}$ values for cytotoxicity of and PEG-TNF- $\alpha$ were $(5246 \pm 85)$ and $(22310 \pm 295)$ IU $\mathrm{mL}^{-1}$, respectively, and both were considerably less cytotoxic than native TNF- $\alpha$ ( $\left.\mathrm{IC}_{50}:(122.45 \pm 3.84) \mathrm{IU} \mathrm{mL}^{-1}\right)$ (Figure 4A). After pulsed (2 h) and long-term (24 h) drug exposure, it was shown that cytotoxic effect of PEGvcTNF- $\alpha$ increased with the length of exposure time, and the growth inhibition rate of long-term PEG-vcTNF- $\alpha$ exposure was higher than that of pulsed term PEG-vcTNF- $\alpha$ exposure, and almost as high (at high concentrations) as that induced by $2 \mathrm{~h}$ treatment with TNF- $\alpha$ (Figure 4B). Cytotoxic effects of PEG-vcTNF- $\alpha$ decreased with CA-074 (10 $\left.\mu \mathrm{mol} \mathrm{L}{ }^{-1}\right)$ and increased with cathepsin B (10 units $\mathrm{mL}^{-1}$ ), compared with the same dose of PEG-vcTNF- $\alpha$ alone. Furthermore, the cytotoxic effects of PEG-vcTNF- $\alpha$ with 10

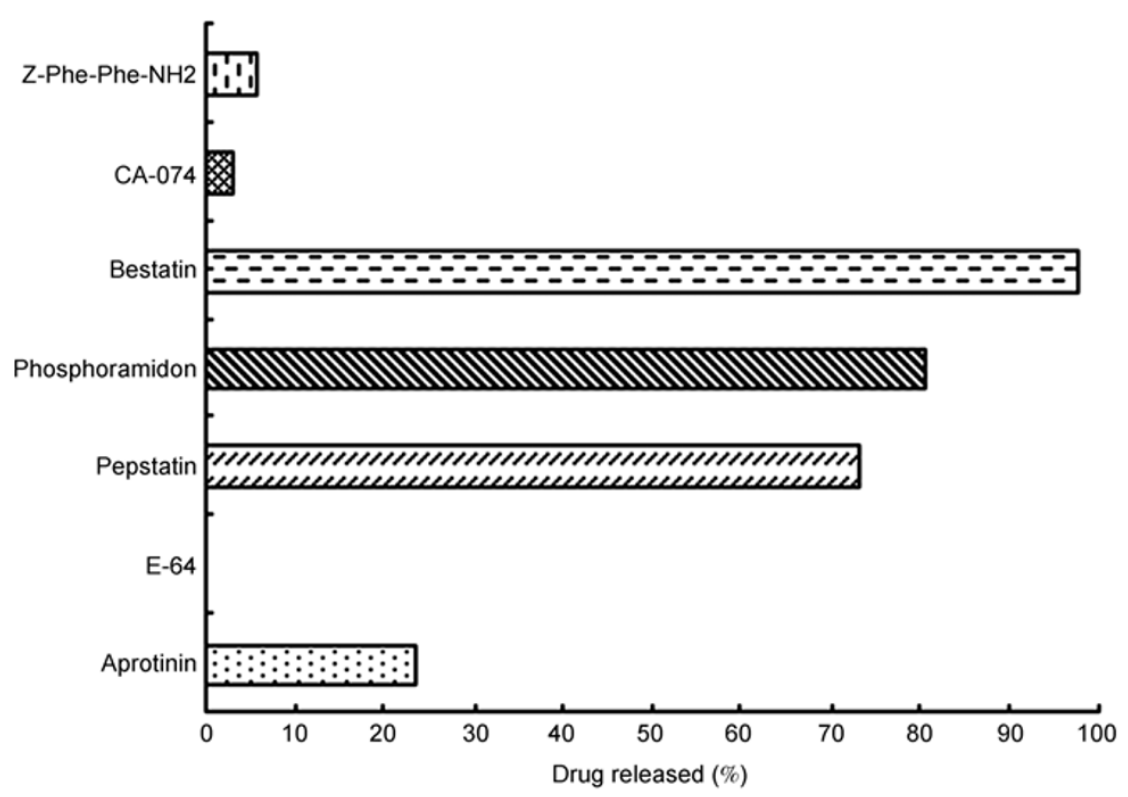

Figure 3 Effect of various protease inhibitors on TNF- $\alpha$ release by incubation with rat liver lysosome extracts. TNF- $\alpha$ release for 24 h is expressed as percentage relative to the control. TNF- $\alpha$ conjugates labeled with FITC, rat liver lysosome, and inhibitor concentrations were approximately $20 \mu \mathrm{g} \mathrm{mL}^{-1}, 0.5$ $\mathrm{mg} \mathrm{mL}^{-1}$ of protein, and $10 \mu \mathrm{mol} \mathrm{L}{ }^{-1}$, respectively. Conditions: $40 \mathrm{mmol} \mathrm{L}^{-1}$ acetate buffer (pH 4.0 ), $1 \mathrm{mmol} \mathrm{L}^{-1} \mathrm{EDTA} 5 \mathrm{mmol} \mathrm{L}^{-1}$ reduced glutathione, $0.1 \%$ Triton $\mathrm{X}-100 ; 37^{\circ} \mathrm{C}$. 

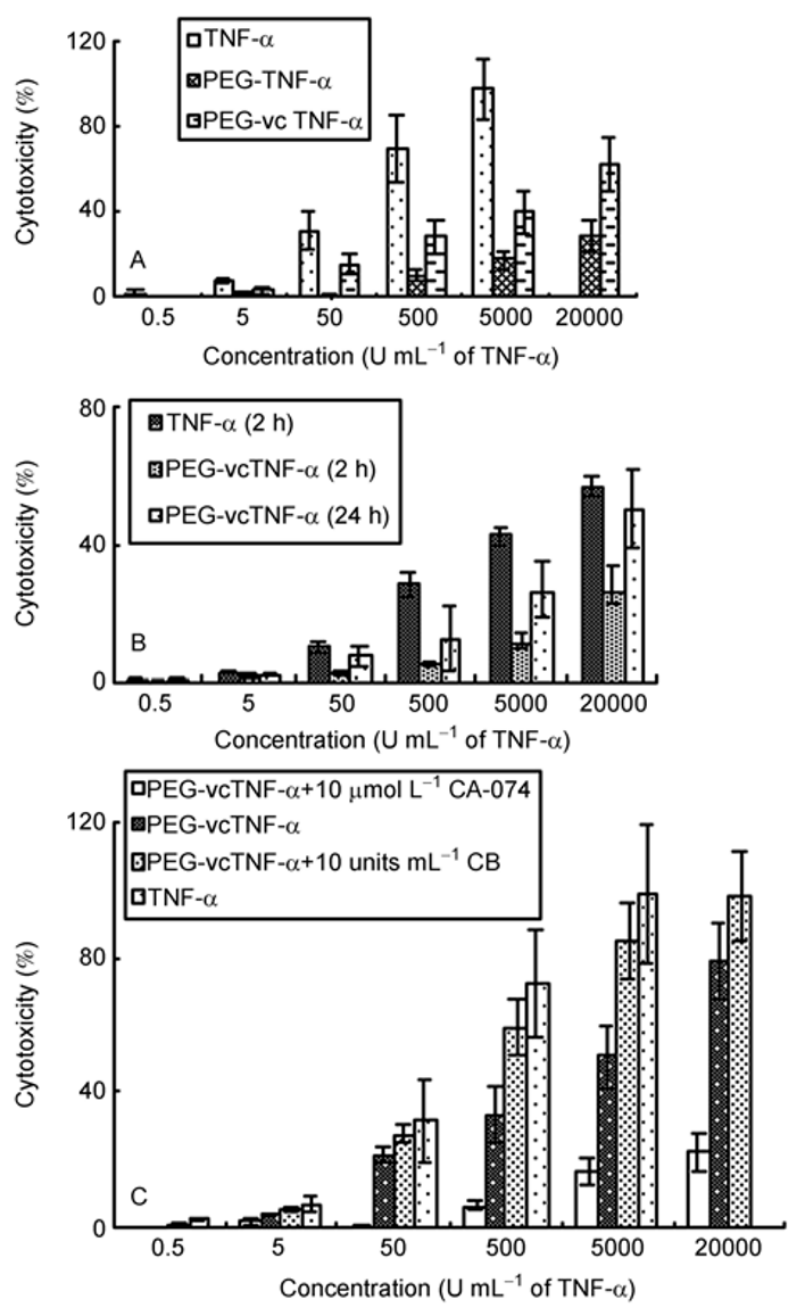

Figure 4 In vitro cytotoxicity of PEG-vcTNF- $\alpha$ and its effect on L929 cell line. CCK- 8 assay was used to measure cytotoxicity. Effects of TNF- $\alpha$, PEG-TNF- $\alpha$ or PEG-vcTNF- $\alpha$ were determined after L929 cells were incubated for $48 \mathrm{~h}$ (A). Shorter incubations ( $2 \mathrm{~h}$ and $24 \mathrm{~h}$ ) were also tested (B). The effects of cathepsin B (10 units $\left.\mathrm{mL}^{-1}\right)$ and CA-074 $\left(10 \mu \mathrm{mol} \mathrm{L} \mathrm{L}^{-1}\right)$ on PEG-vcTNF- $\alpha$ cytotoxicity after a $48 \mathrm{~h}$ incubation were also examined (C). Cytotoxicity (\%) was calculated relative to control culture incubated without TNF- $\alpha$, PEG-TNF- $\alpha$ or PEG-vcTNF- $\alpha$. $n=4$.

units $\mathrm{mL}^{-1}$ cathepsin $\mathrm{B}$ were almost identical to that induced by the same dose of native TNF- $\alpha$ (Figure 4C), suggesting that under these conditions, the entirety of the conjugated drug is being released to act in the same way as native TNF- $\alpha$. To characterize the killing mechanism of L929 cells induced by TNF- $\alpha$, PEG-TNF- $\alpha$ and PEG-vcTNF- $\alpha$, we compared the content of viable, necrotic and apoptotic cells in cell cultures treated for $12 \mathrm{~h}$ with these formulations. As seen in Figure 5A, approximately $83 \%$ of L929 cells in control cultures were viable (bottom left quadrant). Incubation with TNF- $\alpha$ resulted in a large increase in apoptotic cell number (Figure 5B), which was less apparent after incubation with PEG-TNF- $\alpha$ (Figure 5C) and PEG-vcTNF- $\alpha$ (Figure 5D). The number of apoptotic cells decreased when cells were cultured with PEG-vcTNF- $\alpha$ in the presence of CA-074 (Figure 5E), and increased following combined treatment with PEG-vcTNF- $\alpha$ and cathepsin B (Figure 5F). Neither cathepsin B nor CA-074 alone resulted in cell necrosis or apoptosis (data not shown).

\subsection{In vivo study of antitumor effects}

We compared the antitumor potency of PEG-vcTNF- $\alpha$ to those of native TNF- $\alpha$ and PEG-TNF- $\alpha$ with scheduled i.v. injections on Meth-A solid tumors. Control mice (saline or PEG alone) showed no antitumor effect (data not shown), whereas native TNF- $\alpha$ showed suppression of tumor growth (Figure 6). However, three of eight mice administered native TNF- $\alpha$ at a dose of 10000 IU died within $24 \mathrm{~h}$, and the remaining mice developed piloerection, tissue inflammation and a loss in body weight (data not shown). Whereas the minimal dose of native TNF- $\alpha$ (200 IU) was not effective for tumor growth inhibition. PEG-vcTNF- $\alpha$ and PEG-TNF$\alpha$ had markedly increased antitumor potencies compared with native TNF- $\alpha$. As shown in Figure 6, PEG-TNF- $\alpha$ at a dose of 2000 IU showed the maximal antitumor effects without any toxic side effects and had antitumor effects superior to that of native TNF- $\alpha$ at a dose of 10000 IU. On the other hand, only 200 IU of PEG-vcTNF- $\alpha$ was needed to exhibit a marked antitumor potency, and tumor growth was completely inhibited during the observation period. This reversal of tumor growth was only seen at 2000 IU of PEG-TNF- $\alpha$ and at 10000 IU of native TNF- $\alpha$, indicating that PEG-vcTNF- $\alpha$ was a more potent antitumor agent by approximately 10- and 50-fold, respectively, compared to unlinked PEG-TNF- $\alpha$ and native TNF- $\alpha$.

\subsection{Pharmacokinetics studies in mice}

The plasma concentrations of TNF- $\alpha$ after i.v. administration of native TNF- $\alpha$, PEG-TNF- $\alpha$ or PEG-vcTNF- $\alpha$ are shown in Figure 7. Native TNF- $\alpha$ was rapidly eliminated from the circulation, and its plasma half-life was only $0.31 \mathrm{~h}$ (Table 3) which corresponds to that reported previously [32]. In contrast, the plasma clearance of both PEG-TNF- $\alpha$ and PEG-vcTNF- $\alpha$ was markedly decreased relative to that of native TNF- $\alpha$ and their plasma half-lives were 27.23 and $21.22 \mathrm{~h}$, respectively, approximately 85 - and 65-fold longer than that of native TNF- $\alpha$.

\section{Discussion}

The peptide-linked PEGylated protein reported here is distinguished from the general PEGylated proteins described elsewhere in three important aspects: stability in plasma, sensitivity to cathepsin B, and specific activity. Generally, 

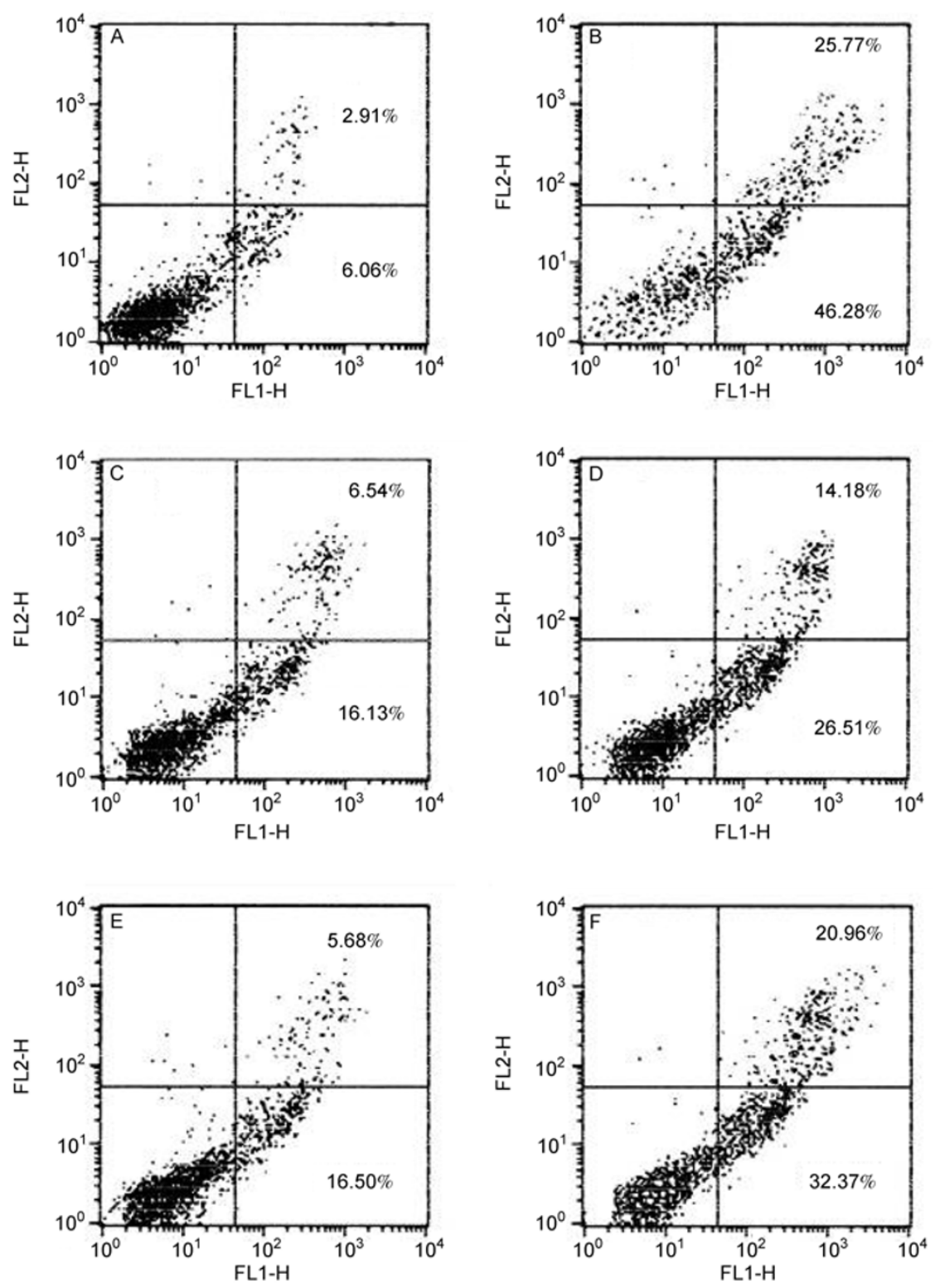

Figure 5 The effect of PEG conjugates on L929 cell apoptosis. L929 cells $\left(1 \times 10^{6}\right.$ cells well $\left.{ }^{-1}\right)$ were plated in multiwell plates. After $24 \mathrm{~h}$, buffer (A), TNF- $\alpha\left(10\right.$ IU mL $\left.{ }^{-1}\right)(B)$, PEG-TNF- $\alpha$ (C), PEG-vcTNF- $\alpha$ (D), PEG-vcTNF- $\alpha$ with CA-074 $\left(10 \mu \mathrm{mol} \mathrm{L}^{-1}\right)$ (e) or PEG-vcTNF- $\alpha$ with cathepsin B (10 units $\left.\mathrm{mL}^{-1}\right)(\mathrm{F})$ was added. Cells were incubated for a further $12 \mathrm{~h}$. Culture medium was then replaced with $100 \mu \mathrm{L}$ cold binding buffer. Cell staining was performed using Annexin V-FITC according to the manufacturer's instructions. Samples were analyzed using a FACSCanto (BD, USA) flow cytometer.

PEGylation of a protein drug enhances its plasma half-life and in vivo stability because of its increased molecular size and steric hindrance, but these improvements are accompanied by a significant loss of their specific activity. In this paper, we describe the use of a cathepsin B-cleavable dipeptide linker in novel PEGylated conjugates to overcome the contradiction between long plasma half-life but low therapeutic efficacy for PEGylated proteins and short half-life/poor tissue selectivity for native TNF- $\alpha$.

PEG polymer is nontoxic and is approved by the FDA for use in drugs, so is used widely to modify therapeutic proteins. Literally thousands of research articles have de- scribed almost every aspect of attachment of PEG to a therapeutic protein. In this work, an N-hydroxysuccinimide derivative of the activated polymer (PEG-NHS or PEG-vcNHS) was used to attach PEG to TNF- $\alpha$. The preparation process of conjugates was according to previously described methods. For PEG-vcTNF- $\alpha$, two amino acids were added to the $\mathrm{N}$ terminal of TNF- $\alpha$ prior to attachment to PEG through the $\mathrm{N}$-hydroxysuccinimide activated ester. We showed that PEG modification had negative effects on the bioactivity of TNF- $\alpha$, which caused reduction in specific activity to between $6.23 \%$ to $74.19 \%$ depending on the degree of modification. For general PEGylation, the remaining 


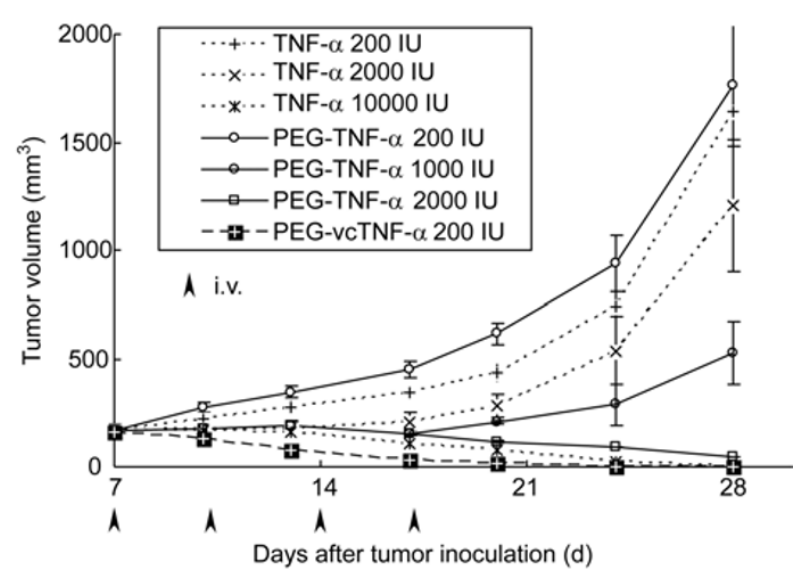

Figure 6 Antitumor effect on Meth-A solid tumors of native TNF- $\alpha$, PEG-TNF- $\alpha$ and PEG-vcTNF- $\alpha$ (at the concentrations shown, per mouse per injection) administered by i.v. injection (at time points indicated by arrows). PEG and saline were used as controls. $n=6$.

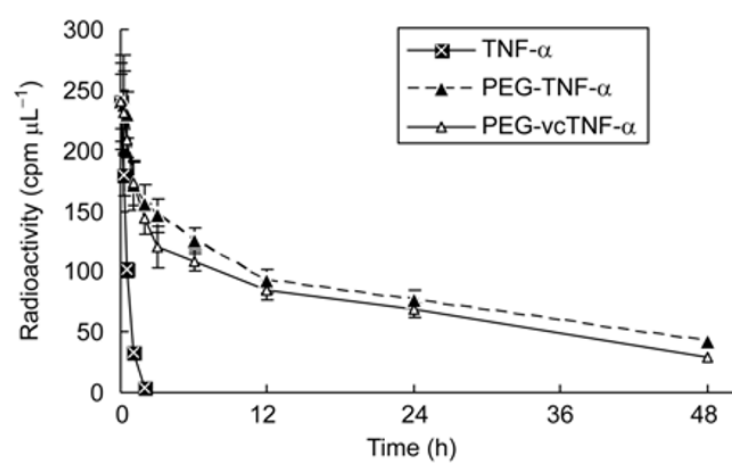

Figure 7 Changes in TNF- $\alpha$ concentration in serum after administration of native TNF- $\alpha$, PEG-TNF- $\alpha$ and PEG-vcTNF- $\alpha$. After i.v. administration of $\left[{ }^{125} \mathrm{I}\right]$-native TNF- $\alpha$ or $\left[{ }^{125} \mathrm{I}\right]$-bioconjugated TNF- $\alpha$ to normal male $\mathrm{BALB} / \mathrm{c}$ mice, blood was collected from tail vein at various time points, and radioactivity was measured. Each bar represents the mean $\pm \operatorname{SD}(n=6)$.

Table 3 Pharmacokinetic parameters of TNF- $\alpha$ in plasma after i.v. administration of different formulations to rats. Data are mean \pm SD, $n=6$

\begin{tabular}{|c|c|c|c|}
\hline Formulation & $t_{1 / 2}(\mathrm{~h})$ & AUC $\left(0\right.$-INF) $\left(\mathrm{cpm} \mu \mathrm{L}^{-1}\right) \mathrm{h}$ & MRT (h) \\
\hline TNF- $\alpha$ & $0.31 \pm 0.03$ & $133 \pm 17$ & $0.44 \pm 0.04$ \\
\hline PEG-TNF- $\alpha$ & $27.23 \pm 5.72$ & $3472 \pm 898$ & $39.29 \pm 8.25$ \\
\hline PEG-vcTNF- $\alpha$ & $21.22 \pm 3.1$ & $3554 \pm 549$ & $30.62 \pm 4.47$ \\
\hline
\end{tabular}

bioactivity of TNF- $\alpha$ decreased as modification degree increased, because of the loss of binding site(s) and/or steric hindrance. This finding suggested that PEG chains sterically hindered the TNF-receptor binding site for PEG-TNF- $\alpha$, consistent with many literature reports. However, when a valine-citrulline moiety was added between PEG and TNF- $\alpha$, we found, unexpectedly, that the loss of bioactivity was much less than without the valine-citrulline linker, indicating that this moiety plays an important role in protecting TNF- $\alpha$ bioactivity.

We hypothesized that TNF- $\alpha$ could be liberated from their conjugates by incubation with proteases. However, released TNF- $\alpha$ will theoretically be degraded by components of the plasma or by the proteases used in these experiments. This prediction is supported by the absence TNF- $\alpha$ in our test conditions (data not shown). Evaluation of PEG conjugate stability in samples taken from plasma or other solutions presents a challenge for drug quantitation, because TNF- $\alpha$ is easily degraded by unspecific proteases in plasma or by lysosomal proteases such as cathepsin B. When TNF- $\alpha$ is released from PEG conjugates into plasma, it is cleared very quickly, so the free drug was undetectable (data not shown). Accordingly, it is very difficult to directly quantify released TNF- $\alpha$ in the presence of plasma or proteases. In this study, PEG is used as an amphichroic molecule, such that when PEG attaches to a protein, that protein becomes similarly amphichroic. Making use of the amphi- chroic features of PEG or PEG conjugates, TNF- $\alpha$ conjugates labeled with FITC or PEG in plasma samples were extracted with chloroform [26]. Fluorescence intensity was then analyzed with a fluorescence spectrophotometer. TNF- $\alpha$ concentration (including conjugates) can be validated by to this process, which was highly reproducible (data not shown).

As shown in Figure 1, significant drug loss occurs in plasma, possibly because of the presence of unspecific proteases in mouse and human plasma [33]. However, the stability of TNF- $\alpha$ in both PEG conjugates in plasma was similar, indicating that the degradation mostly results from the direct effect of these proteases on the TNF- $\alpha$ rather than on the cathepsin B-sensitive peptide linker (valine-citrulline). The cathepsin B-sensitive peptide linker therefore seems resistant to protease actions while in the circulation. It was found that the half-life of drug loss for PEG-vcTNF- $\alpha$ is more than five days when incubated in human plasma, confirming that the dipeptide linkers used in PEG-vcTNF- $\alpha$ offer a significant stability advantage in human plasma, which is crucial for the in vivo efficacy of the conjugate. Drug loss from both conjugates was faster when incubated in human plasma, suggesting that more protease activity is present in human plasma than in mouse plasma. This finding was consistent with the report that human plasma has much higher levels of protease activity than mouse plasma [33]. 
Knowledge of enzyme specificity permits the design of drug-polymer conjugates that can be activated specifically at the tumor site. Cathepsin B is an attractive target for the release of drugs, because increases in the levels of mRNA, protein, enzyme activity, membrane association, and secretion of cathepsin B have all been observed in tumors. Therefore, cathepsin B-specific drug release observed in the case of PEGylated protein is thought to be advantageous from the viewpoint of presenting the cytotoxic agent selectively at the tumor site. We showed that PEG-vcTNF- $\alpha$ was cleaved by cysteine proteases, but not other proteases, to release TNF- $\alpha$, and this release is dependent upon $\mathrm{pH}$. Our finding that the valine-citrulline linker peptide is specifically targeted by cysteine proteases to release the peptide drug from the PEGylated conjugate is in agreement with previous reports [20-24].

Here the question arises of whether cathepsin B liberates TNF- $\alpha$ within the lysosomes or outside the cell. The answer is to be found in our observations that the release of TNF- $\alpha$ relies on $\mathrm{pH}$, with the optimum condition being $\mathrm{pH} 4$. With regard to $\mathrm{pH}$, tumors were thought for many years to have a more acidic $\mathrm{pH}$ than most normal tissues. The major difference lies in the extracellular $\mathrm{pH}$. In normal tissue extracellular $\mathrm{pH}$ is at about 7.4, whereas in many tumors it is about $0.3-0.5 \mathrm{pH}$ units more acidic in comparison to intracellular $\mathrm{pH}$ ( $\mathrm{pH}$ 7.1-7.2) [34]. This extracellular acidification in tumors is insufficient for the release of TNF- $\alpha$, indicating that drug release occurs in the lysosomes.

To further prove that it is cathepsin B that acts on the peptide linker, the effect of several enzyme inhibitors was evaluated, including aprotinin, bestatin, pepstatin, phosphoramidon, E-64, Z-Phe-Phe-CHN 2 , and CA-074. In Figure 3, E-64, CA-074 and Z-Phe-Phe- $\mathrm{CHN}_{2}$ were all able to dramatically suppress the release. E-64 is a non-specific inhibitor of cysteine proteases, while CA-074 and Z-PhePhe- $\mathrm{CHN}_{2}$ are specific inhibitors of cathepsin B and cathepsin L, respectively. This suggests that lysosomal cathepsins $\mathrm{B}$ and $\mathrm{L}$ are responsible for the cleavage and further support our earlier results.

The L929 cell was used in these tests because this cell line is particularly sensitive to TNF- $\alpha$. It was proven again at the cellular level that PEGylation of proteins is accompanied by a significant loss of their activities. However, we show in Figures 4 and 5 that PEG-vcTNF- $\alpha$ induced higher cytotoxicity and more apoptosis in these cells than PEGTNF- $\alpha$, through the mechanism of cathepsin-specific release of TNF- $\alpha$, as demonstrated by the enhancement and inhibition of this response by cathepsin $\mathrm{B}$ and the cathepsin B inhibitor, CA-074, respectively. Thus, the dipeptide linkers used appear to play an important role on the improvement of in vivo antitumor efficacy of PEG-vcTNF- $\alpha$. Cytotoxicity of PEG-vcTNF- $\alpha$ was observed to be both doseand time-dependent. Although very low, PEG-TNF- $\alpha$ showed some cytotoxicity, possibly because of incomplete modification by PEG.

The antitumor effect of PEG-vcTNF- $\alpha$ compared with PEG-TNF- $\alpha$ and native TNF- $\alpha$ was improved greatly, which might reflect their improved plasma stability, circulation time, tumor selectivity etc. The poor antitumor effect of native TNF- $\alpha$ indicates its fast elimination from the plasma, while the improved efficacy of PEG-vcTNF- $\alpha$ suggests that more drug is released in the tumor tissues. In comparison with native TNF- $\alpha$, the antitumor effect of PEG conjugates increased significantly, about 50-fold for PEG-vcTNF- $\alpha$ and 5-fold for PEG-TNF- $\alpha$, respectively. As shown in Figure 6, PEG has no toxicity when administered intravenously at this dose and has not shown antitumor effects in tumor-bearing mice. Thus, it is the PEGylation of TNF- $\alpha$, not the PEG itself, which contributes to the improvements in pharmacodynamics. The shielding of proteolytic cleavage sites of TNF- $\alpha$ by the PEG chain and reduction of the renal clearance due to increased molecular size lead to the increase in circulation time and plasma stability for both PEG-TNF- $\alpha$ and PEG-vcTNF- $\alpha$, as demonstrated in the our pharmacokinetic and in vitro stability studies. Additionally, it is well known that macromolecules are accumulated and retained in the tumor tissue much more than in normal tissues, a phenomenon termed the "enhanced permeability and retention (EPR)" effect [35,36]. Many macromolecular anticancer agents, such as synthetic polymer-conjugating drugs, polymeric micelle-containing drugs and others, have been reported accordingly [32,37]. The increased tumor growth inhibition seen here with the TNF- $\alpha$ conjugates might also result from the EPR effect of PEGylation.

However, with the same degree of PEG modification, PEG-vcTNF- $\alpha$ was approximately 10 -fold more potent than PEG-TNF- $\alpha$, suggesting that the linkage with the cathepsin B-sensitive valine-citrulline dipeptide as a cleavable moiety further promotes the anticancer activity of the PEGylated TNF- $\alpha$ against murine fibrosarcoma. Although the inserted dipeptide is important for the selective release of drugs from the conjugate in tumor tissue, it is necessary to recognize the contribution of PEGylation per se in conferring increases in circulation time, plasma stability, and the EPR effect. Thus, it is crucial to combine both PEG and a protease-sensitive linker to achieve better anticancer activity. We have demonstrated here the in vivo mechanism of action of PEG-vcTNF- $\alpha$ after administration by i.v. injection. PEGvcTNF- $\alpha$ has a longer circulation time than TNF- $\alpha$, and accumulates and is retained in the tumor tissue because of the EPR effect. Once sequestered in this way, PEG-vcTNF- $\alpha$ is cleaved and TNF- $\alpha$ is released from the dipeptide PEG conjugates through enzyme hydrolysis in the tumor tissue, where cathepsin B was expressed extracellularly. This leads to high local concentrations of TNF- $\alpha$ in the tumor tissue, which greatly enhances it anticancer efficacy. We believe that this technology is a major advance in the delivery of 
protein anti-cancer agents to their intended site of action, and will hopefully lead to improved therapies in the fight against cancer.

This work was supported by the National High Technology Research and Development Program of China (Grant No. 2007AA021811), the National Natural Science Foundation of China (Grant No. 30701055) and Postdoctoral Science Foundation of China (Grant No. 20070410029). We would also like to thank Professor Lu Wei, Peking University, for assistance in data analysis with WinNonlin.

1 Carswell E A, Old L J, Kassel R L, et al. An endotoxin-induced serum factor that causes necrosis of tumors. Proc Natl Acad Sci USA, 1975, 72: 3666-3670

2 Debs R J, Fuchs H J, Philip R, et al. Immunomodulatory and toxic effects of free and liposome-encapsulated tumor necrosis factor alpha in rats. Cancer Res, 1990, 50: 375-380

3 Leist M, Jaattela M. Four deaths and a funeral: From caspases to alternative mechanisms. Nat Rev Mol Cell Biol, 2001, 2: 589-598

4 Blick M, Sherwin S A, Rosenblum M, et al. Phase I study of recombinant tumor necrosis factor in cancer patients. Cancer Res, 1987, 47: 2986-2989

5 Creaven P J, Plager J E, Dupere S, et al. Phase I clinical trial of recombinant human tumor necrosis factor. Cancer Chemoth Pharm, 1987, 20: 137-144

6 Kimura K, Taguchi T, Urushizaki I, et al. Phase I study of recombinant human tumor necrosis factor. Cancer Chemoth Pharm, 1987, 20: 223-229

7 Sherman M L, Spriggs D R, Arthur K A, et al. Recombinant human tumor necrosis factor administered as a five-day continuous infusion in cancer patients: phase I toxicity and effects on lipid metabolism. J Clin Oncol, 1988, 6: 344-350

8 Chapman P B, Lester T J, Casper E S, et al. Clinical pharmacology of recombinant human tumor necrosis factor in patients with advanced cancer. J Clin Oncol, 1987, 5: 1942-1951

9 Rosenberg S A, Lotze M T, Muul L M, et al. A progress report on the treatment of 157 patients with advanced cancer using lymphokineactivated killer cells and interleukin-2 or high-dose interleukin-2 alone. New Engl J Med, 1987, 316: 889-897

10 Caliceti P, Veronese F M. Pharmacokinetic and biodistribution properties of poly(ethylene glycol)-protein conjugates. Adv Drug Deliv Rev, 2003, 55: 1261-1277

11 Wang Y S, Youngster S, Grace M, et al. Structural and biological characterization of PEGylated recombinant interferon alpha-2b and its therapeutic implications. Adv Drug Deliv Rev, 2002, 54: 547-570

12 Kozlowski A, Harris J M. Improvements in protein PEGylation: PEGylated interferons for treatment of hepatitis C. J Control Release, 2001, 72: 217-224

13 Yoshioka Y, Tsutsumi Y, Ikemizu S, et al. Optimal site-specific PEGylation of mutant TNF- $\alpha$ improves its antitumor potency. Biochem Bioph Res Co, 2004, 315: 808-814

14 Yamamoto Y, Tsutsumi Y, Yoshioka Y, et al. Site-specific PEGylation of a lysine-deficient TNF-alpha with full bioactivity. Nat Biotech, 2003, 21: 546-552

15 Wu X, Li X, Zeng Y, et al. Site-directed PEGylation of human basic fibroblast growth factor. Protein Expres Purif, 2006, 48: 24-27

16 Otto H H, Schirmeister T. Cysteine proteases and their inhibitors. Chem Rev 1997, 97: 133-172

17 Devetzi M, Scorilas A, Tsiambas E, et al. Cathepsin B protein levels in endometrial cancer: Potential value as a tumour biomarker. Gynecol Oncol, 2009, 112: 531-536

18 Hashimoto Y, Kakegawa H, Narita Y, et al. Significance of cathepsin
B accumulation in synovial fluid of rheumatoid arthritis. Biochem Bioph Res Co, 2001, 283: 334-339

19 Barrett A J, Kembhavi A A, Brown M A, et al. L-trans-Epoxysuccinylleucylamido (4-guanidino) butane (E-64) and its analogues as inhibitors of cysteine proteases including cathepsins B, H and L. Biochem J, 1982, 201: 189-198

20 Francisco J A, Cerveny C G, Meyer D L, et al. cAC10-vcMMAE, an anti-CD30-monomethyl auristatin E conjugate with potent and selective antitumor activity. Blood, 2003, 102: 1458-1465

21 Afar D E H, Bhaskar V, Ibsen E, et al. Preclinical validation of anti-TMEFF2-auristatin E-conjugated antibodies in the treatment of prostate cancer. Mol Cancer Ther, 2004, 3: 921-932

22 Dubowchik G M, Firestone R A, Padilla L, et al. Cathepsin B-labile dipeptide linkers for lysosomal release of doxorubicin from internalizing immunoconjugates: model studies of enzymatic drug release and antigen-specific in vitro anticancer activity. Bioconjug Chem, 2002, 13: 855-869

23 Sanderson R J, Hering M A, James S F, et al. In vivo drug-linker stability of an anti-CD30 dipeptide-linked auristatin immunoconjugate. Clin Cancer Res, 2005, 11: 843-852

24 Doronina S O, Toki B E, Torgov M Y, et al. Development of potent monoclonal antibody auristatin conjugates for cancer therapy. Nat Biotech, 2003, 21: 778-784

25 Ajaj K A, Biniossek M L, Kratz F. Development of protein-binding bifunctional linkers for a new generation of dual-acting prodrugs. Bioconjug Chem, 2009, 20: 390-396

26 Stocks S J, Jones A J M, Ramey C W, et al. A fluorometric assay of the degree of modification of protein primary amines with polyethylene glycol. Anal Biochem, 1986, 154: 232-234

27 Yamazaki S, Onishi E, Enami K, et al. Proposal of standardized methods and reference for assaying recombinant human tumor necrosis factor. Jpn J Med Sci Biol, 1986, 39: 105-118

28 Faure M P, Gaudreau P, Shaw I, et al. Synthesis of a biologically active fluorescent probe for labeling neurotensin receptors. J Biol Chem, 1994, 42: 755-763

29 Aniento F, Roche E, Cuervo A M, et al. Uptake and degradation of glyceraldehyde-3-phosphate dehydrogenase by rat liver lysosomes. J Biol Chem, 1993, 268: 10463-10470

30 Murata M, Miyashita S, Yokoo C, et al. Novel epoxysuccinyl peptides. Selective inhibitors of cathepsin B, in vitro. FEBS Lett, 1991, 280: 307-310

31 Barrett A J, Kirschke H. Cathepsin B, Cathepsin H, and Cathepsin L. Methods Enzymol, 1981, 80: 535-561

32 Tsutsumi Y, Kihira T, Tsunoda S, et al. Molecular design of hybrid tumor necrosis factor-alpha III: Polyethylene glycol-modified tumor necrosis factor-alpha has markedly enhanced antitumor potency due to longer plasma half-life and higher tumor accumulation. J Pharmacol Exp Ther, 1996, 278: 1006-1011

33 Satoh T, Hosokawa M. The mammalian carboxylesterases: From molecules to functions. Annu Rev Pharmacol Toxicol, 1998, 38: 257-288

34 Vaupel P, Kallinowski F, Okunieff P. Blood flow, oxygen consumption and tissue oxygenation of human tumors. Adv Exp Med Biol, 1990, 277: 895-905

35 Mahajan S S, Hou L, Doneanu C, et al. Optimization of bivalent glutathione S-transferase inhibitors by combinatorial linker design. J Am Chem Soc, 2006, 128, 8615-8625

36 Vasey P A, Kaye S B, Morrison R, et al. Phase I clinical and pharmacokinetic study of PK1 (HPMA copolymer doxorubicin): First member of a new class of chemotherapeutic agents-drug-polymer conjugates. Clin Cancer Res, 1999, 5: 83-94

37 Yokoyama M, Okano T, Sakurai Y, et al. Toxicity and antitumor activity against solid tumors of micelle-forming polymeric anticancer drug and its extremely long circulation in blood. Cancer Res, 1991, 51: $3229-3236$

Open Access This article is distributed under the terms of the Creative Commons Attribution License which permits any use, distribution, and reproduction in any medium, provided the original author(s) and source are credited. 